\title{
ABBIAMO PUBBLICATO NEGLI ULTIMI NUMERI
}

\begin{abstract}
N. 25/2009
Come conciliare la solidarietà sociale e l'efficienza economica nell'era della globalizzazione: un punto di vista "regolazionista" di Robert Boyer Le condizioni contrattuali e previdenziali del lavoro degli anziani: politiche e interventi di attuazione di Marco Accorinti e Paolo Calza Bini Anzianità attiva e salute in Europa: il progetto ActivAge di Sandro Turcio e Paolo Calza Bini Mercato del lavoro e settore cooperativo in Italia di Andrea Bernardi e Pasquale Tridico Dall'urbanistica al governo del territorio: i nuovi orientamenti della pianificazione e delle politiche urbane di Lorenzo Ranzato
\end{abstract}

\section{N. 26/2009}

Gruppo Europeo di Cooperazione Territoriale (GECT). Prove di coesione territoriale di Gianluca Spinaci L'Europa antagonista e riformatrice di Andrea Pierucci e Giancarlo Vitella Lo Stato spagnolo delle autonomie e l'integrazione europea di Francesc Morata Cluster e distretti tecnologici: modelli e politiche di Salvatore Monni e Alessandro Spaventa La politica dei cluster in Europa: tendenze e criticità emergenti di Alessandro Minello

\section{N. 27/2009}

L'Occidente e il Resto del Mondo nell'economia mondiale: un'interpretazione Maddisoniana e Malthusiana dal 1000 al 2030 di Angus Maddison La programmazione regionale in Italia: una rassegna della letteratura economica di Aurelio Bruzzo Intermodalità e interportualità nel sistema di trasporto merci in Italia di Maurizio Mistri Infrastrutture e compensazioni: considerazioni a partire dalle evidenze empiriche di Fulvio Adobati e Vittorio Ferri Sistemi produttivi locali e competizione globale. Analisi di due regioni industriali: la produzione legno-arredo in North Carolina e nel Nord-Est italiano di Giulio Buciuni

\section{N. 28/2010}

Il finanziamento delle piccole e medie imprese e la crisi finanziaria: un quadro di riferimento di Gregory F. Udell Il risparmio e i suoi nemici di Mauro Marconi Le misure di politica economica per le PMI nelle ZFU italiane: opportunità e problematiche di Aurelio Bruzzo Un'analisi delle normative regionali per il coordinamento dei tempi della città di Alessandra Fasano I commercianti e l’immigrazione straniera: accoglienza e diffidenza di Massimiliano Crisci 


\section{N. 29/2010}

Fonti di finanziamento per le nuove Imprese Tecnologiche: un'indagine sulle imprese della Fondazione Kauffman di Alicia Robb Sviluppo locale e obiettivi della programmazione di Sebastiano Fadda Il livello della qualità della vita: questioni territoriali di Aurelio Bruzzo Welfare e sviluppo locale di Giovanni Bertin Interconnessioni tra sviluppo economico e demografico nel declino urbano: il caso di Genova di Paolo Calza Bini, Caterina Cortese, Alberto Violante

\section{N. 30/2011}

Elementi per un cambiamento delle relazioni tra le imprese italiane di Ilario Favaretto Le istituzioni economiche: chiave per comprendere e per superare la crisi di Sebastiano Fadda Processi cognitivi ed organizzazione dei distretti industriali di Maurizio Mistri L'economia italiana nei sentieri del capitalismo imprenditoriale di Giancarlo Corò L'intervento pubblico a livello regionale: suo failure e nuova formulazione? di Aurelio Bruzzo Quale sviluppo? Quale locale? Ripensando i sistemi territoriali nel terzo millennio di Paolo Calza Bini Potrà l'Europa avere una politica all'altezza della "prospettiva 2020"? di Franco Sotte

N. 31/2011

Aggregazione di imprese e contratto di rete di Giuseppe Travaglini La (s)composizione della flessibilità lavorativa: modulazione, forma e organizzazione del lavoro di Emiliano Mandrone La criminalità organizzata quale intermediario nel mercato del lavoro di Antonella Mennella Crisi economica, criminalità e vincoli di liquidità delle imprese di Ilario Favaretto e Germana Giombini La decrescita serena secondo Serge Latouche. Critica di un economista istituzionalista di Maurizio Mistri

\section{N. 32/2011}

Lo scambio talent for citizenship e la politica europea per l'immigrazione ad alta qualificazione di Gabriele Orcalli Noi e gli altri: l'atteggiamento degli italiani nei confronti degli immigrati di Vincenzo Memoli Il lavoro artigiano nella globalizzazione di Stefano Micelli I distretti nella crisi: declino, adattamento o innovazione? di Alessia Busato e Giancarlo Corò Progettare il processo costituente di un cluster. Il caso del distretto trentino dell'energia e dell'ambiente di Paolo Gurisatti 